\title{
Prise en charge des fractures mandibulaires à l'hôpital Saint Luc de Cotonou (Bénin). A propos de 83 cas
}

\author{
Sylvie Arlette Bancolé Pognon ${ }^{1,}{ }^{*}$, Imrane Biotchane ${ }^{2}$, Georges Dossou Akpovi ${ }^{3}$ \\ ${ }^{1}$ Service de Stomatologie, Centre national hospitalier universitaire, Cotonou, Bénin \\ ${ }^{2}$ Service d'ORL et de Chirurgie cervico-faciale, Centre national hospitalier universitaire, Cotonou, Bénin \\ 3 Hôpital St Luc, Cotonou, Bénin
}

(Reçu le 31 décembre 2012, accepté le 11 février 2013)

Mots clés :

fractures

mandibulaires /

épidémiologie /

thérapeutique

\begin{abstract}
Résumé - Il s'agit d'une étude descriptive rétrospective sur les fractures mandibulaires traitées dans le Service de Chirurgie de l'hôpital Saint Luc de Cotonou (Bénin) de juin 2006 à décembre 2009. Le but de ce travail était d'évaluer la prise en charge des fractures mandibulaires.

Au total, 83 cas de fractures mandibulaires ont été répertoriés. L'âge moyen des patients était de 30,16 $\pm 10,16$ ans avec une fourchette allant de 6 à 72 ans. Le sex ratio H/F était de 8,33. L'étiologie était dominée par les accidents de la voie publique ( $85 \%$ des cas). La région parasymphysaire constituait la principale localisation $(35,71 \%$ des cas), souvent associée à d'autres localisations dans le cadre de fractures complexes (39\% des cas). Les méthodes thérapeutiques étaient dominées par le traitement chirurgical (ostéosynthèse) et tous les patients ont été traités sous anesthésie générale.
\end{abstract}

Key words: mandibular fractures / epidemiology / treatment

\begin{abstract}
Support for mandibular fractures in Saint Luc hospital of Cotonou (Benin): 83 cases report. This is a retrospective descriptive study on the mandibular fractures treated in the surgical department of Saint Luc Hospital of Cotonou (Benin) between june 2006 and december 2009. The purpose of this study was to assess the support of mandibular fractures in Saint Luc hospital of Cotonou (Benin). This study has identified 83 cases of mandibular fractures. The average age was $30.16 \pm 10.16$ years with a range from 6 to 72 years. The sex ratio H/F was 8.33. The etiologie was dominated by the accidents of the public road ( $85 \%$ of cases). In the anatomopathologic fields, the parasymphysis came top in the locations ( $35.71 \%$ of cases), with associations determinant of fractures often complex (39\% of cases). The therapeutic methods were dominated by the surgical treatment (osteosynthesis) and all patients had been treated under general anesthesia.
\end{abstract}

La mandibule est une structure unique, médiane, symétrique et mobile [1]. Elle constitue à elle seule le massif facial inférieur [2]. A la suite de traumatismes d'origines diverses, elle peut présenter des fractures de formes variable. Les fractures de la mandibule constituent une solution de continuité complète ou incomplète, avec ou sans déplacement des fragments osseux. En termes de fréquence, elles représentent les $2 / 3$ des fractures du massif facial [1].

L'intérêt de cette étude réside dans le fait qu'il y a un seul chirurgien maxillo-facial qui exerce dans le Service de Chirurgie cervico-faciale du Centre national hospitalier et universitaire (CNHU) de Cotonou (Bénin), insuffisamment équipé en matériels pour la traumatologie maxillo-faciale. Tous les cas de traumatologie maxillo-faciale sont orientés vers l'hôpital Saint Luc de Cotonou (Bénin).

L'objectif de cette étude était d'évaluer la prise en charge des fractures mandibulaires en vue de faire des suggestions pour l'amélioration du plateau technique du Service de Chirurgie cervico-faciale du CNHU.

\section{Patients et méthode}

Il s'agit d'une étude descriptive rétrospective sur la période de juin 2006 à décembre 2009 qui a eu pour cadre le Service de Chirurgie de l'hôpital Saint Luc de Cotonou (Bénin), hôpital de $1^{\text {er }}$ niveau de référence. Le registre du bloc opératoire a été

\footnotetext{
*Correspondance : pobasfr@yahoo.fr
} 


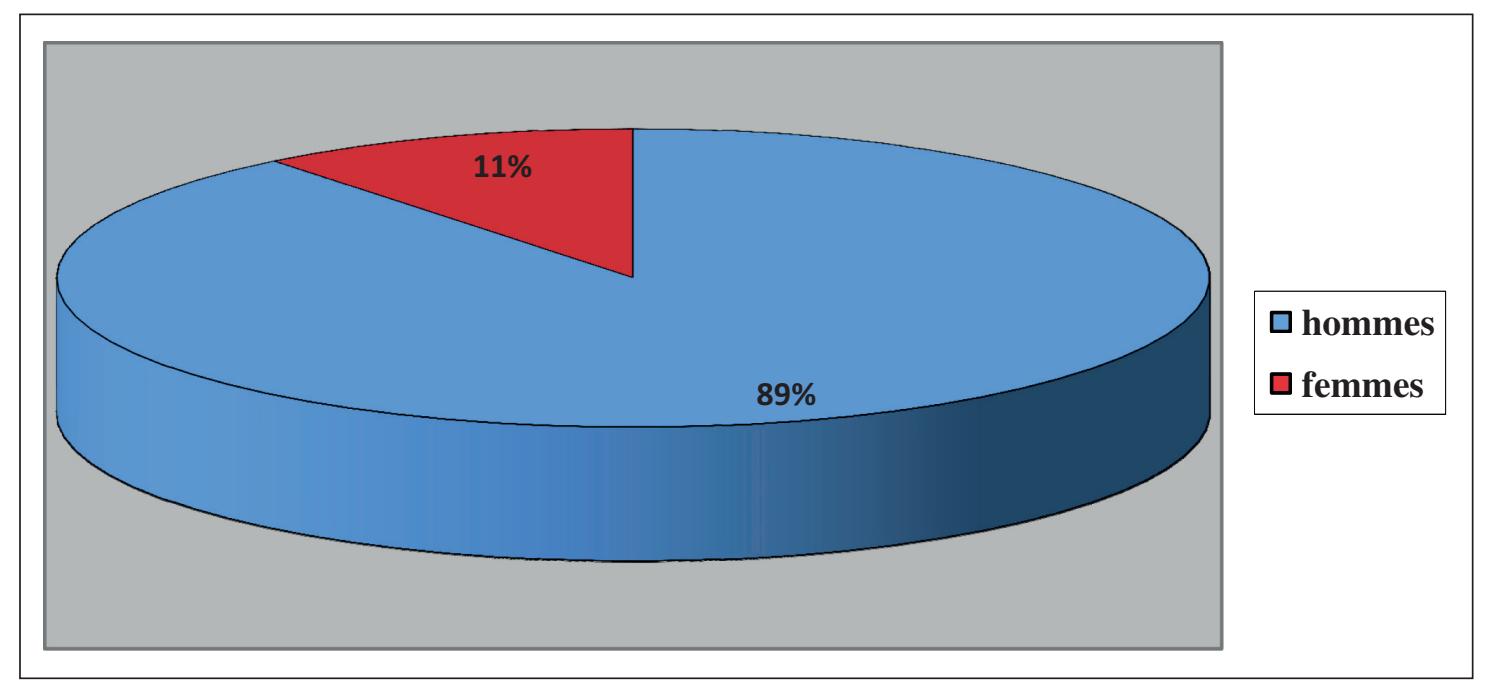

Fig. 1. Répartition selon le sexe.

Fig. 1. Distribution according to the sex.

exploité et les dossiers de fracture mandibulaire avec diagnostic clinique et radiologique et traitement chirurgical et/ou orthopédique ont été retenus.

L'âge, le sexe, l'étiologie, le siège de la fracture, le délai de consultation et la durée d'hospitalisation représentent les variables exploitées. La saisie et l'analyse des données ont été faites grâce au logiciel STATA version 11 .

\section{Résultats}

\section{Données épidémiologiques}

\section{Fréquence}

Au total, 2375 actes chirurgicaux ont été réalisés durant la période de l'étude. $7 \%$ correspondaient à des cas de traumatismes maxillo-faciaux, 50,3\% de ces derniers soit 83 cas étant des fractures mandibulaires.

\section{Répartition selon l'âge et le sexe}

La fourchette d'âge des patients était comprise entre 6 et 72 ans. L'âge moyen était de $30,16 \pm 10,16$ ans ; $85 \%$ des cas compris dans la tranche d'âge de 20-39 ans et la tranche d'âge la plus représentée était celle de $30-39$ ans avec $48 \%$ des cas.

La Figure 1 montre la répartition selon le sexe. 89,29\% des cas étaient de sexe masculin, soit un sex ratio $\mathrm{H} / \mathrm{F}$ de 8,33.

\section{Répartition selon l'étiologie}

Les accidents de la voie publique (AVP) représentaient $84,93 \%$ des cas. L'étiologie était significativement liée au
Tableau I. Répartition selon l'étiologie. Table I. Distribution according to the aetiology.

\begin{tabular}{lcc}
\hline Étiologie & Cas & $\%$ \\
\hline Accident de sport & 2 & 2,74 \\
Accident domestique & 1 & 1,37 \\
Agression & 2 & 2,74 \\
AVP & 62 & 84,93 \\
Avulsion dentaire & 1 & 1,37 \\
Chute & 2 & 2,74 \\
Coups et blessures volontaires & 3 & 4,11 \\
\hline Total & 73 & 100,00 \\
\hline
\end{tabular}

sexe avec $p=0,001$; en effet, les accidents domestiques et les agressions étaient plus en cause chez les sujets de sexe féminin alors que les AVP, les accidents de sport et les coups et blessures volontaires l'étaient plutôt chez les hommes. Le Tableau I montre la répartition selon l'étiologie.

\section{Aspects cliniques}

Dans $64,28 \%$ des cas, les fractures concernaient la région parasymphyse-symphyse seule ou associée à une autre région de la mandibule ; les fractures des branches horizontales arrivaient en $2^{\mathrm{e}}$ position avec $29,76 \%$, suivies de celles de l'angle $(19,04 \%)$, puis de celles de la région condylienne $(3,57 \%)$. Les fractures alvéolo-dentaires représentaient $4,76 \%$; dans $39 \%$ des cas ces fractures étaient associées.

La répartition selon le foyer de fracture présentée dans la Figure 2 montre que dans $54 \%$ des cas les fractures étaient unifocales, $39 \%$ bifocales. 


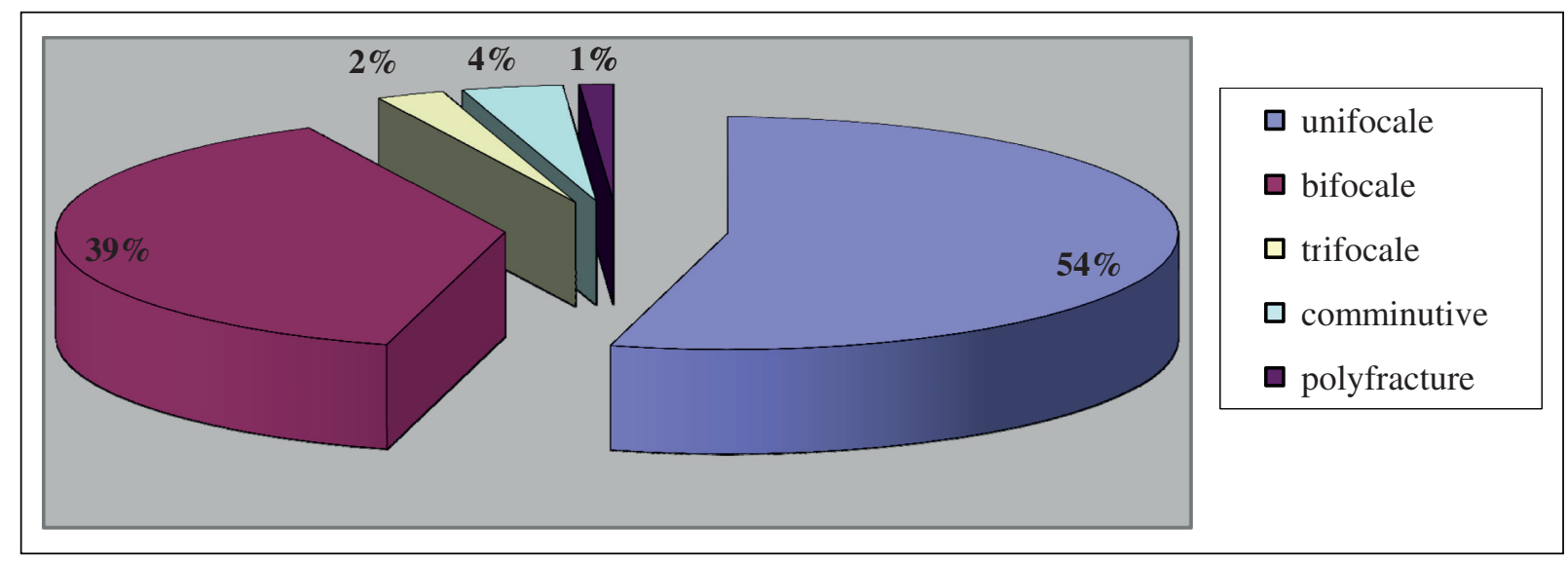

Fig. 2. Répartition selon le nombre de foyers fracturaires.

Fig. 2. Distribution depending on the number of fracture sites.

Tableau II. Répartition selon la thérapeutique utilisée.

Table II. Distribution according to the therapeutic used.

\begin{tabular}{lcc}
\hline Type de traitement & Cas & 1,22 \\
\hline Arc unimaxillaire & 1 & 15,85 \\
Blocage intermaxillaire/arcs & 13 & 7,32 \\
Blocage intermaxillaire/ligatures d'Ivy & 6 & 1,22 \\
Fils intraosseux & 1 & 1,22 \\
Fils intraosseux + blocage intermaxillaire/Ivy & 1 & 30,49 \\
Plaques vissées & 25 & 18,29 \\
Plaques vissées + blocage intermaxillaire/arcs & 15 & 6,10 \\
Plaques vissées + blocage intermaxillaire/arcs et Ivy & 5 & 14,63 \\
Plaques vissées + blocage intermaxillaire/Ivy & 12 & 2,44 \\
Plaques vissées + fils intraosseux & 2 & 1,22 \\
Plaques vissées + fils intraosseux + blocage intermaxillaire/Ivy & 1 & 100,00 \\
\hline Total & 82 & \\
\hline
\end{tabular}

\section{Aspects thérapeutiques}

Une ostéosynthèse a été réalisée dans $71 \%$ des cas et $23 \%$ des cas ont bénéficié d'un traitement orthopédique par blocage inter-maxillaire. Tous les patients ont été traités sous anesthésie générale. Le Tableau II présente la répartition selon le type de traitement. Sur 61 dossiers, la durée moyenne d'hospitalisation était de $9 \mathrm{j} \pm 5 \mathrm{j}$ (3-35 j). Sur 58 dossiers, le délai moyen d'attente avant traitement était de 13 jours.

\section{Commentaires et discussion}

\section{Aspects socio-démographiques}

Fréquence

Dans cette étude, un traumatisme maxillo-facial sur deux était associé à une fracture mandibulaire. Ces chiffres sont 
similaires à ceux rapportés par d'autres auteurs avec une fréquence de fractures mandibulaires supérieure à $50 \%$ [3-6]. Cette fréquence élevée est probablement liée à la situation anatomique de la mandibule et à sa fonction de pare-chocs pour la face.

\section{Âge et sexe}

L'âge moyen des patients était de $30 \pm 10$ ans et $85 \%$ des cas avaient un âge compris dans la tranche d'âge 20-39 ans et le sex ratio $\mathrm{H} / \mathrm{F}$ était de 8,33.

Ces résultats sont superposables à ceux d'études antérieures réalisées aussi bien en Afrique $[3,7,9,12,13]$ qu'au Pakistan [8] ou au Canada $[10,11]$ : l'âge moyen des patients était de $30 \pm 6$ ans, le sex ratio $H / F \geq 4$ et plus de $50 \%$ des cas avaient entre 20 et 30 ans. Ces sujets jeunes sont en général très actifs donc vulnérables aux traumatismes. Les fractures mandibulaires pourraient constituer un frein à la productivité et au développement des pays car elles occasionnent des incapacités temporaires de travail.

\section{Étiologie}

Dans cette étude, l'étiologie était dominée par les AVP (85\% des cas), puis venaient les violences interpersonnelles $(6,7 \%$ des cas) et les accidents de sport ( $5 \%$ des cas). Ces résultats sont superposables à ceux donnés par les travaux antérieurs [4].

Au Bénin les AVP seraient favorisés:

- Le non respect du code de la route et le défaut du port de casque et de ceinture de sécurité par les usagers de la route ;

- le nombre important de taxis motos conduisant sans permis de conduire ;

- l'usage des substances addictives (alcool et drogues) ;

- la vétusté du parc automobile privé et la légèreté du contrôle technique au centre national de sécurité routière.

Les mêmes causes produisant certainement les mêmes effets, la situation n'est pas différente dans les autres pays en développement: les AVP constituent la principale cause de fractures mandibulaires $[7,8,12,13]$ sauf à Madagascar où ce sont les violences interpersonnelles [3]. Dans les pays développés, les violences interpersonnelles représentent la principale cause de fractures mandibulaires (41 à $57 \%$ des cas), les AVP seulement 6 à $28 \%$ des cas $[10,11,14]$.

Malgré la densité du parc automobile et du trafic routier dans les pays développés, les AVP ne constituent pas la principale étiologie. Cette situation serait certainement due aux différentes politiques de sensibilisation des populations au respect du code de la route et aux diverses mesures de répression des écarts de comportements dans la circulation (retrait de permis aux contrevenants, limitation de vitesse, répression de la conduite en état d'ivresse, etc.). Les facilités d'acquisition de véhicules neufs dans ces pays et les possibilités de dépannage de problèmes techniques seraient certainement des facteurs de réduction des AVP dus aux défaillances techniques. C'est là un modèle que les pays en développement gagneraient énormément à répliquer pour réduire les traumatismes dus aux AVP.

Par contre, dans les pays développés, les actes de violences représente la principale étiologie des fractures mandibulaires ; c'est sans doute le corollaire du développement où une frange non négligeable de la population, marginalisée pour de multiples raisons, trouve dans la violence un moyen d'expression. Il est à noter que les pays en développement ne sont plus épargnés par cette vague de violences interpersonnelles, surtout dans les grandes villes. Par exemple à Antananarivo (Madagascar) [3], $56 \%$ des fractures mandibulaires sont secondaires à des violences interpersonnelles, probablement pour les mêmes raisons que dans les pays développés.

\section{Siège du trait de fracture}

La région parasymphysaire représente la principale localisation des traits de fracture $(35,71 \%$ des cas $)$. Globalement, il y a concordance avec les résultats de travaux antérieurs quant à la localisation parasymphyso-symphysaire du trait de fracture (27 à $55 \%$ des cas selon les études) [8, 11, 13]. Il existe probablement une corrélation entre cette localisation parasymphyso-symphysaire de la fracture et les AVP où le choc se fait plus volontiers sur le menton selon un impact direct.

La prédominance par ordre de fréquence décroissante du trait de fracture au niveau du condyle (36\%), de la branche horizontale $(21 \%)$ et de l'angle $(20 \%)$, associée à une fréquence élevée de violences interpersonnelles, rapportée par $d^{\prime}$ autres auteurs $[4,5]$, pourrait faire penser à une éventuelle corrélation entre cette étiologie et ces localisations avec un impact direct (angles et branches horizontales) ou indirect (condyle).

\section{Nombre de foyers de fracture}

Dans les 83 cas rapportés, la fracture mandibulaire était unifocale dans $54 \%$ des cas et bifocales dans $39 \%$ des cas. Ces chiffres ne sont pas différents de ceux retrouvés dans la littérature : la fréquence des fractures unifocales est toujours supérieures ou égales à $50 \%$ des cas et celle des fractures bifocales varie de 25 à $44 \%$ des cas $[3,9,12,14]$.

\section{Aspects thérapeutiques}

Pour ces 83 cas, l'intervention chirurgicale a toujours été réalisée sous anesthésie générale et, dans $71 \%$ des cas, on a effectué une ostéosynthèse. Ces pourcentages sont 
comparables à ceux de Rocton et al. (ostéosynthèse dans $73 \%$ des cas mais ces cas ont été traités à Paris) [14]. Par contre, dans les études réalisées dans les pays africains, le traitement orthopédique représente le traitement de première intention [7, 12]. Pour Quevauvilliers et al., le blocage intermaxillaire est souvent suffisant pour traiter les fractures sans déplacement et l'ostéosynthèse indiquée pour les fractures déplacées [15]. En conséquence, tout ou partie des $54 \%$ des fractures unifocales auraient pu être traitées de façon orthopédique et les $23 \%$ de blocage intermaxillaire auraient pu se faire sous anesthésie locale. L'anesthésie générale ayant un coût et des risques ; elle devrait être réservée aux cas complexes ne pouvant être traités sous anesthésie locale ou loco-régionale.

Les choix thérapeutiques devraient normalement être établis en fonction du type fracture et du niveau de développement du pays. Au Bénin, le défaut de structures d'approvisionnement en matériels d'ostéosynthèse limite sérieusement l'accessibilité à la technique chirurgicale. Certes, l'ostéosynthèse procure un certain confort per- opératoire au chirurgien et postopératoire au patient mais cet argument ne suffit pas à justifier sa généralisation. De plus, les victimes de traumatismes maxillo-faciaux sont souvent démunies, ce qui pose un problème d'accessibilité financière aux soins. Une alternative aux plaques vissées serait l'ostéosynthèse aux fils d'acier mais les risques liés à l'anesthésie générale demeurent.

\section{Conclusion}

La prolifération des taxis motos au Bénin est une des causes de la recrudescence des accidents de la voie publique avec comme corollaire les fractures mandibulaires. Ces fractures qui représentent plus de la moitié des traumatismes maxillo-faciaux intéressent plus l'adulte jeune de sexe masculin. Elles constituent un réel problème de santé publique.

Le traitement orthopédique est suffisant dans la plupart des cas pour la consolidation des fragments. Le matériel technique indispensable n'étant pas très onéreux à acquérir, le CNHU est invité à dynamiser le Service de Chirurgie cervicofaciale en le dotant de matériels de traumatologie maxillofaciale. De même, les autorités du Ministère de la santé sont invitées à former et recruter des spécialistes en chirurgie buccale et maxillo-faciale.

Conflits d'intérêt : aucun

\section{Références}

1. Lezy JP, Princ G. Pathologie maxillo-faciale et stomatologie (pp. 26-37). Masson, Paris, 2004.

2. Collège hospitalo-universitaire français de chirurgie maxillofaciale et stomatologie. 3- Coordination Lebeau J. Chirurgie maxillo-faciale et stomatologie pour le $2^{\mathrm{e}}$ cycle des études médicales (pp. 51-4). Elsevier Masson, Paris, 2009.

3. Razafindrabe JAB, Rakotoarisoa AHN, Rakoto FA, Randriamanantenasoa VH, Rakotozafy LF, Rakotovao JD. Épidémiologie des fractures de la mandibule traitées au Centre hospitalier universitaire d'Antananarivo-Madagascar. Rev Trop Chir 2007;1:33-5.

4. Zagury G, Bruyère F. MédiChiffres : les 8000 chiffres-clés de la médecine clinique de (p. 258). Édition Estem, Paris, 1998.

5. Carli P, Riou B, Telion C. Urgences médico-chirurgicales de l'adulte (pp. 604-5). Arnette, Paris, 2004.

6. Bouguila J, Zairi I, Khonsari R H, Jablaoui Y, Hellali M, Adouani A. Épidémiologie de la traumatologie maxillofaciale à Tunis. Rev Stomatol Chir Maxillofac 2008;109:353-7.

7. Bouguila J, Zairi I, Khonsari R H, Lankriet C, Mokhtar M, Adouani A. Particularités épidémiologiques et thérapeutiques des fractures de mandibule au CHU Charles-Nicolle de Tunis. Rev Chir Maxillofac 2009;110:81-5.

8. Khan A, Salam A, Khitab U, Khan M T. Pattern of mandibular fractures - A Study. Pak Oral Dent J 2009;29:221-4.

9. El Khatib K, Gradel J, Danino A, Mouaffak M, Malka G. Alimentation entérale par sonde naso-gastrique : intérêt après ostéosynthèse des fractures mandibulaires. Rev Stomatol Chir Maxillofac 2005;106:13-5.

10. Sojat AJ, Meisami T, Sandor GKB, Clokie CML. Epidémiologie des fractures de la mandibule traitées à l'hôpital général de Toronto : revue de 246 cas. J Can Dent Assoc 2001;67:640-4.

11. Czerwinski M, Parker WL, Chehade A, Williams HB. Identification of mandibular fracture epidemiology in Canada: enhancing injury prevention and patient evaluation. Can J Plast Surg 2008;16:36-40.

12. Ngouoni B G, Mathey-Manza, Moyikoua. Résultats du traitement des fractures mandibulaires. À propos de 169 cas. Méd Afr Noire 1996;43:529-32.

13. Dia Tine $S$, Tamba B, Niang P, Gassama Barry C, Kébé NF, Guéye NF, Guéye I, Diallo B. Fractures de la mandibule en pratique odontologique : à propos de 103 cas. Méd Buccale Chir Buccale 2009;15:137-45.

14. Rocton S, Chaine A, Ernenwein D, Bertolus C, Rigolet A, Bretrand JC, Ruhin B. Fractures de la mandibule : épidémiologie, prise en charge thérapeutique et complications d'une série de 563 cas. Rev Stomatol Chir Maxillofac 2007;108:3-10.

15. Quevauvilliers J, Perlemuter G, Perlemuter L. Dictionnaire médical de l'infirmière : l'encyclopédie pratique de référence (p. 414) $8^{\mathrm{e}}$ éd. Elsevier Masson, Paris, 2009. 